\title{
La actividad censal en España durante la primera mitad del siglo XIX en el contexto de las reformas liberales
}

\author{
Antonia M. ${ }^{\text {a }}$ DURÁn HERRERA * \\ Doctoranda. UNED
}

\section{Census activities in Spain during the first half of the nineteenth century within the context liberal changes}

\begin{abstract}
RESUMEN
España, durante la primera mitad del siglo xix, vive un proceso de modernización caracterizado por la transición hacia un régimen liberal constitucional. La realización del censo era primordial, las guerras civiles e inestabilidades políticas impidieron su ejecución. A través del sistema de Imputaciones, hubo una información constante del volumen de población. Las imputaciones nos revelan la ocultación sistemática de la población,

la trascendencia de las modificaciones administrativas-territoriales y una aproximación a los distintos ritmos de crecimiento. Aunque no hubo censos generales, desde 1833 hasta 1856, se crearon las bases del desarrollo legislativo

$e$ institucional de todas las actividades censales del siglo XIX.
\end{abstract}

PALABRAS CLAVE: Palabras claves: imputaciones, censos, población, regiones, provincias, estadísticas, catastro, normativas.

\section{ABSTRACT}

During the first midhalf of the nineteenthcentury, Spain lived a modernisation process looking forward a constitutional and liberal political regime. To making a demographic census was always a goal never attained by reason of civil wars and political instability. In order to know on the ground the real population, the Government developed a special population statistics, named Imputations. They reveal us the concealment of the real population, they are meaningfully available for understanding the territorial and administrative changes, and we can get an approximate idea of the rate of growth and the regional distribution population. Even though, there is not a general census between 1833-1856, a lot of rules were given in order to create special institutions, and a general census structure for all 19th century.

\section{KEYWORDS:}

Imputations, census, population, regional, provinces, statistics, rules

* Este artículo es uno de los capítulos del trabajo de Investigación El Lenguaje de los censos en el proceso de modernización de España. Departamento de Historia Contemporánea. U.N.E.D. 2006. La autora es Catedrática de Geografía e Historia. I.E.S. Bárbara de Braganza. Badajoz. 


\section{INTRODUCCIÓN}

El desarrollo de las actividades censales, durante la primera mitad del siglo XIX, viene determinados por los acontecimientos políticos internacionales, como la invasión francesa en España, el proceso de descomposición del antiguo régimen en Europa, los nuevos planteamientos político-administrativos del liberalismo, la nueva estructuración social, las desamortizaciones, las transformaciones económicas derivadas de la industrialización, y la propia dinámica científico- racionalista con la creación de los Institutos de Estadísticas y de Estudios Geográficos. Estas realidades se convirtieron en factores consustanciales del nuevo sistema censal. El siglo se inició con la publicación en 1803 del Censo de Godoy-Larruga, realizado entre los años 1799-1801, como: "Censo de Frutos y Manufacturas de España e islas adyacentes, ordenado sobre los datos dirigidos por los Intendentes y aumentados con las principales....»'.

Por avatares de la vida política no se pudo realizar otro censo general hasta 1857. Siguiendo un orden cronológico, las actividades conducentes a la elaboración de censos demográficos se podrían sintetizar en varios ejes, que a modo de vectores, siguiendo la teoría de sistemas ${ }^{2}$, se convierten en principios generadores de la transformación general que operó en el ámbito administrativo, social y en particular, en la renovación de los censos electorales y catastros fiscales.

En el contexto sociopolítico de modernización, el objetivo era la creación de un estado moderno, racional, donde el principio de igualdad jurídica de los ciudadanos ante la ley quedaba recogido en todas las constituciones; la estadística censal individualizada es un elemento fundamental para la administración, tanto desde el punto de vista fiscal y social como de la consideración de la fortaleza y prestigio de una nación en función del número de sus habitantes y reflejo de un estado constitucional liberal. El volumen de población era garantía de poder fáctico en el ámbito internacional, como se advierte en las noticias internacionales de la Gaceta oficial de Madrid.

\section{REFORMAS ADMINISTRATIVAS, TERRITORIALES Y CENSALES.}

Por el R.D. del 25 de septiembre de 1799, se habían creado seis nuevas provincias $^{3}$ que modificaban el censo de Floridablanca; era el inicio de un proceso de transformación jurídico-territorial que duraría hasta 1833. Las implicaciones de los cambios territoriales en la organización administrativa eran básicas en cuanto se modificaba la «naturaleza de los habitantes del territorio»; los antiguos reinos,

\footnotetext{
1 Así se denomina la primera publicación oficial del censo de Godoy-Larruga. Madrid. 1801. Edición facsímile. I.N.E. Madrid. 1992

2 Bertalanffy, L. Teoría general de los sistemas. México. FCE. 7. a edic. 1989

3 Bargueño Rivero, J. Geografía política de la España constitucional. La división provincial. Madrid. Centro de Estudios Constitucionales. 1996.
} 
principados, provincias, abadengos, etc. pasaron a ser provincias. Esta uniformidad territorial-administrativa va directamente relacionada con el número de habitantes, rentas y catastro, levantamientos topográficos, datos agrícolas, ganaderos y fundamentalmente, repercutía en el número de ciudadanos que podían tener acceso al voto electoral.

En 1808, bajo el gobierno de José I, el coronel Fransec Amorós presentó, acorde con los requerimientos de Napoleón de establecer un sistema territorial-administrativo común al francés, una división territorial en departamentos que no se puso en práctica por los acontecimientos de Bailén 4 . Siguiendo el proyecto del clérigo Llorente que proponía 38 prefecturas y 111 subprefecturas, D. Mariano Luis de Urquijo dispuso que "se dividirá España, para el Gobierno Civil, en veinticuatro Prefecturas, cuyas capitales son... $»^{5}$.

Las Cortes de Cádiz, al aprobar la Constitución de 1812, proyectaron la España peninsular en 18 provincias, a las que se añadían los territorios en América y Asia6:

«... Aragón, Asturias, Castilla la Nueva, Cataluña, Córdoba, Extremadura, Galicia, Granada, Jaén, León, Murcia, Navarra, Provincias Vascongadas, Sevilla y Valencia, islas Baleares y las Canarias. En la América septentrional Nueva España, con la Nueva Galicia, Guatemala, provincias internas de Oriente, provincias internas del Occidente, isla de Cuba, con las dos Floridas, la parte española de la isla de Santo Domingo y la isla de Puerto Rico, con las demás adyacentes y al continente en uno y otro mar. En la América meridional la Nueva Granada, Venezuela, el Perú, Chile, provincias del Río de la Plata y todas las islas adyacentes en el mar Pacífico y en el Atlántico. En Asia las islas Filipinas y las que dependan de su gobierno».

En esta constitución, el concepto de «español», no es un simple epónimo, sino que la mentalidad de los liberales, lo hacía intrínseco a una nación, cuyos límites sobrepasaban los «lindes» de Europa, afectando a los territorios de América, África y Asia. Las poblaciones allí residentes fueron consideradas españolas y por tanto, dejaban de ser "coloniales», para ser ciudadanos incluidos en el censo.

En 1813, Felipe Bauzá diseñó una nueva división territorial con 44 provincias. Con el regreso de Fernando VII en 1814, la anulación de la Constitución de 1812 y los Decretos del 4 de Mayo de 1814, se paralizaron todas las reformas ${ }^{7}$ iniciadas, volviendo a la situación político-administrativa característica del antiguo régimen.

Durante el Trienio Constitucional, el gobierno liberal retomó la actividad reformista y llevó a cabo la propuesta de organización territorial, con la formación de 52

\footnotetext{
${ }^{4}$ La derrota francesa de Bailón dio un lugar al abandono de Madrid por parte de José I y su ocupación posterior por Napoleón. En los años de máximo dominio francés, 1809-1810, se replanteó la reforma territorial.

${ }^{5}$ El Decreto 17 Abril de 1810 firmado por L. Urquijo. Cfr. Bargueño. Op. Cit. 1996.

${ }^{6}$ Art. 12. "Constitución de Cádiz, 1812. GonZÁlez MuÑIS, M. A. Constituciones, Cortes y Elecciones españolas. Historia y anécdota. (1910-1936).Gijón. Ediciones Júcar, 1978.

7 Decretos del 4 de Mayo de 1814. G.M. 12 de mayo 1814. Se anularon todas las reformas llevadas a cabo por las Cortes de Cádiz.
} 
provincias y el diseño de nuevos límites provinciales ${ }^{8}$. La finalización trágica del trienio en 1823, la nueva anulación de la Constitución, hizo que dicha división no se llevara a efecto.

Al morir Fernando VII (29-IX-1833), dejaba como sucesora a su hija Isabel (1833-1868) de tres años de edad, por lo que se nombra a su madre, M. ${ }^{\text {a }}$ Cristina de Borbón, Regente y Gobernadora(1833-1840) ${ }^{9}$. En ese mismo año de 1833, Javier de Burgos(1778-1849) ${ }^{10}$, Secretario de Fomento, dispuso la nueva división territorial siguiendo un modelo de administración funcional, de tipo centralista, el resultado fue la organizaron de las 49 provincias. Un año después, (R.D. 18-4-1834), se organizaron las subdivisiones provinciales en partidos judiciales y municipios, fijándose una población como cabeza de partido. Se modificaron las competencias de los Alcaldes, que dejaron de tener potestad judicial ${ }^{11}$, pasando a tener funciones ejecutivas, formando parte de las Juntas censales. Esta estructura territorial-administrativa ha sido la base de organización de todos los censos.

\section{EVOLUCIÓN DE LA ESTADÍSTICA Y LOS SERVICIOS CATASTRALES.}

Las Cortes de Cádiz ${ }^{12}$, a imitación de Francia, aprobaron una disposición (23VI-1813) por la que se aprobaba el plan para formar la Estadística en Sevilla, dirigido por Flores Estrada ${ }^{13}$, como institución encargada de la elaboración del censo.

8 Gaceta de Madrid. 12 de Enero de 1822. Inexplicablemente, la fecha del decreto es la misma que la de la Gaceta. Fernando Saravia (Galicia) y Antonio González Ron (León) proponían una división en 38 provincias.

9 Fernando VII había declarado la vigencia de la Pragmática dada por Carlos IV en las Cortes de 1788. Los acontecimientos políticos de 1832 le hicieron anular dicha ley; el 30 de junio de 1833 reunidas las Cortes en la iglesia de San Jerónimo, se reconoció a la princesa Isabel, de tres años de edad, como legítima heredera.

10 Burgos, J. Anales del Reinado de Isabel II. Madrid. Tipografía Mellado. 1850-51. 6 v. Desde París, el 24 de enero de 1826 había dirigido a Fernando VII un panegírico sobre los males que afectaban a España y los posibles remedios; muerto el rey, se hace cargo de la reforma administrativa.

11 R.D. 18-4-1834. G.M. 23/4/1834. Art.3. Establece que las competencias judiciales que hasta ese momento desempeñaban los alcaldes ordinarios, pasaran a los jueces, cabezas de partido; los Corregidores y Alcaldes mayores de cabeza de partido seguirían administrando justicia, hasta nuevas resoluciones. Era la base institucional de la elaboración de los censos y responsabilidades del funcionariado.

12 Flores Estrada, A. Plan para formar la Estadística en la provincia de Sevilla. Sevilla 1814. Madrid. Edición facsímile del I.N.E. 1983. En Francia, Napoleón había creado en 1800 el Boureau d-Éstatisquié, aunque no llevó a efecto ningún censo, continuó existiendo con la restauración: R. LE MÉE. « La statisque démographique officielle 1815-1870 en France». en Annales de démograsphie historique. École des Hautes Estudes en Sciencies Sociales. Paris. 1979. pp. 251- 279.

13 Flores Estrada, A. ( 1765-1853) fue encargado por las Cortes para organizar el Plan para formar la Estadística en Sevilla. Diferenciando 7 secciones. Agricultura y tierra; ganados; casas y edificio población; industria y comercio; festividades; educación. Publicó en dos tomos un curso de Economía política. Partidario de una desamortización diferente a la propuesta por Mendizábal, comprendía la importancia de un censo que precediera a la desamortización.

SÁnCHEZ-LAfUENTE. Historia de la estadística como ciencia (1500-1900). Madrid. Ministerio de Planificación y Desarrollo. 1975. pág. 107 y ss. 
Las necesidades económicas obligaban, y la natural evolución cultural-científica coadyuvaban, a la elaboración de instrumentos básicos de información fiscal y geográfica. Por el R.D. de 30 de mayo de 1817, Martín de Garay (1771-1822), ${ }^{14}$ creó la Estadística General de España, dirigida por los Intendentes provinciales con un carácter eminentemente fiscal. Los decretos e instrucciones dados para elaborar los censos, no se realizaron, pero tuvieron como efecto el levantamiento de planos topográficos y la elaboración de la Carta geográfica de España. Población y territorio era coordenadas básicas para la organización de un Estado que trataba de suprimir los derechos jurisdiccionales señoriales.

Tras la muerte de Fernando VII, la necesidad de llevar a cabo un censo fiable, no sólo con sentido fiscal o catastral, derivado del proceso desamortizador ${ }^{15}$, era una necesidad imperiosa para la elaboración del censo electoral, con posibilidades de ser aplicada a cualquier campo de la administración ${ }^{16}$. Entre 1833 y 1856 se desarrollaron un creciente número de disposiciones legales que perfilaron progresivamente el sistema de recogida de datos, desde la base más elemental, pasando por las instituciones jerárquicas más altas, en consonancia con los acuerdos jurídicos-diplomáticos del momento, hasta quedar definitivamente constituida la Comisión de Estadística ${ }^{17}$. A pesar de los decretos de 1835, 1836 y $1837^{18}$, el censo general de población no llegó a hacerse. Aprobada y promulgada la Constitución de 1837, el Real Decreto del 29-6-1837, daba las instrucciones correspondientes para formar "los estados o censos de las provincias» ${ }^{19}$. La guerra carlista, problemas de inestabilidad política, levantamiento de los liberales progresistas, la ausencia de una organización administrativa adecuada en provincias y falta de recursos humanos y materiales, hicieron imposible el censo.

14 Garay, Martín de, Memoria presentada por Don Martín de Garay, Ministro del despacho de Hacienda, al Rey. Madrid. 1817

15 Las Leyes Desamortizadoras de Mendizábal (1835/1836) y de Madoz (1855), hacían imprescindible el conocimiento de habitantes, del catastro y de la riqueza agrícola y ganadera de la nación. Se entiende perfectamente que Madoz desarrolle una intensa actividad estadística y censal.

${ }_{16}$ El desarrollo de la actividad estadística de esta época está recogida en SAMPAIO, A.P.F. Elementos de la Ciencia estadística. Madrid, 1841.Edición facsímile. INE. Madrid. 1982

17 En 1843 D. Pascual MADOZ, encargado de Hacienda, nombra la Comisión de Estadística para que capitalice todos los trabajos diseminados y se inicia el proyecto de Ley para la creación de un organismo específico. GACETA DE MADRID: En 1846: 14 normas de regulación de este servicio; en 1847: 11 normas reguladores; en 1848: 18 normas, instrucciones, órdenes diversas de nombramientos, funciones, competencias de dicha institución; 1850: 6 órdenes, decretos; 1851: 6 órdenes; 1852: 2; $1853: 6$ reales decretos; 1854: 4 reales decretos, 2 circulares y en 1855: 7 Reales órdenes que delimitan las funciones y separan sistemas estadísticos.

18 Decreto 23-7-1835 G.M./ 24/7/1835. Reorganización de Ayuntamientos, alcaldes y regidores. Las sesiones de Cortes del 18 al 21 de Enero de 1836, centradas en la discusión de la ley electoral, implicaban indirectamente la elaboración de censos. La R. O de 19-I-1836 (G.M. 21-I-1836)completa la anterior; el R.D. 21 de Agosto de 1836. (G.M. 23 de Agosto) elaboración del censo electoral.

19 R.D. 29-7-1837 (G.M.1-Julio-1837, n. ${ }^{\circ}$ 942) por el que se ordenaba la elaboración del Censo y se daban Instrucciones muy precisas para su elaboración, empadronamiento; modelos y tipos de relación domiciliaria, etc., catalogación social de la población; normas que debían seguir los comisionados; Clasificación de los habitantes por profesión, edad y estado y presentación del estado de la población por provincia. 
Durante la Regencia del general Espartero (1841-1843) las imputaciones de población tuvieron una clara finalidad fiscal y electoral; por el Decreto del $7 / 2 / 1841$ se crearon las juntas de contabilidad en pueblos, en partidos judiciales y en provincias

«La estadística es la piedra angular de toda administración paternal y justa. Sin ella es imposible realizar las mejoras materiales que el país necesita; no cabe equidad en la distribución de las cargas ni beneficios públicos, ni es dado legislar con cierto ni gobernar en paz los pueblos"... ${ }^{20}$.

La preocupación por el conocimiento real de la población se mantuvo: en 1841 se mandó establecer el registro de población en todas las capitales, cabezas de partido y poblaciones mayores de 500 vecinos $^{21}$; en 1846 se publicaron 14 normas reguladoras de la comisión estadística, en 1847 se dieron 11; 18 órdenes en 1848; 6 órdenes e instrucciones en 1850 y así sucesivamente.

El largo gobierno de los liberales moderados (1843-1854), mantuvo el sistema de las imputaciones para la elaboración del censo electoral2 ${ }^{22}$. A partir de 1856, con la vuelta de los moderados al poder, la normativa referida al censo viene imbricada en los organismos institucionales de carácter político, económico e internacional. Hasta la elaboración del censo oficial de 1857, contamos con otras fuentes de información: las oficiales, unida a la normativa que afecta a la organización de Ayuntamientos, las informaciones parroquiales y las informaciones geográficas con relación a los catastros. Durante todo el año de 1856 fueron numerosas las ordenes para elaborar un censo general:

- Ley de Ayuntamientos (5 de julio 1856) por el que se han de formar la Estadística y los Padrones de sus distritos ${ }^{23}$, se crean, se suprimen o se agregan Ayuntamientos y términos y se hacen obligatorios los libros del Registro Civil.

- Instrucciones dadas a los Administradores de la Hacienda Pública y Diputaciones provincial para la formación de las Estadísticas territorial y pecuaria e instrucciones a la Dirección General de Contribuciones, para la formación de la Estadística de contribución industrial y de comercio ${ }^{24}$.

- El R.D. de 24 de Octubre determina que bajo la jurisdicción del Ministerio de Gracia y Justicia, se abra un Negociado de Estadística General del Clero ${ }^{25}$.

20 Decreto del 7/2/1841. G. M. 9/2/1841: N. ${ }^{\circ} 2305$.

21 R. D. 13 de enero 1841.G. M.14/17/1841

22 R.D. 10-3-1845. (G.M. 12 de marzo 1845, n. ${ }^{\circ}$ 3832). Ley electoral en la que se inserta un censo de población.

${ }^{23}$ Ley de Organización y Administración Municipal. G.M. 6 de Julio. N. ${ }^{\circ} 1280$.

24 Amplia legislación durante los meses de Julio de 1856 referida a la normalización de los sistemas contributivos en los que se referencia el cómputo de población. No es un censo propiamente dicho, sino referencias catastrales. G. M. Julio-Agosto de 1856.

25 R. D. 24 de Octubre. G. M. 25-octubre-1856. n. ${ }^{\circ}$ 1391. Censo específico del personal eclesiástico. Durante el gobierno Moderado de Bravo Murillo se firmó el Concordato con la Santa Sede. Opuestos al 
La actividad legisladora de este periodo concluía con los RR.DD. del 3 y 27 de noviembre de $1856^{26}$, ordenando la Comisión de Estadística General del Reino y aprobando el Reglamento de la Comisión de Estadística, organizada en torno a cuatro ejes: la sección primera estaba relacionada con servicios geográficos; la sección segunda referida a "censo general de población", con las condiciones sociales de los habitantes del reino y el movimiento de la población ${ }^{27}$; las secciones tercera y cuarta referidas al catastro y a la contabilidad de rentas y empresas del Estado. A partir de este momento, el sistema censal quedaba organizado en: Censo general de la población, Fuerzas militares de mar y tierra, Estadística de Instrucción Pública, Estadística de Beneficencia, Estadística criminal, Estadística de costumbres públicas y estadística de seguridad.

Paralelamente, en Europa se desarrollaba una labor similar: en Francia, el Bureau de Statisque conseguía en 1836 el primer censo general, reflejando aspectos ya recogidos en el censo de Floridablanca; posteriormente, en 1852 se produjo la reorganización de los Sevices statisque que inspiraron las reformas españolas ${ }^{28}$. En 1853, en Bruselas, Quetelet inició el desarrollo de la estadística moderna al fundar la asociación "Congrés Internacional de Statistique», seguido de la reunión Internacional de Londres de 1860. En ese contexto, dentro de los cometidos del Ministerio de Fomento, se organizó el censo español de 1857.

De este modo, durante la primera mitad del siglo XIX nos encontramos con la creación de un cuerpo legislativo y unas reformas político-administrativas que, si de momento no se aplican por la inestabilidad del sistema político, los cambios de gobierno y por la guerra carlista, tendrán como consecuencia la creación de unas instituciones y actividades censales con fines electorales, económicos, tributarios y puramente demográficos. Se había iniciado la estadística moderna.

\section{LA REALIDAD DE LAS ACTIVIDADES CENSALES OFICIALES.}

Durante el siglo XIX, las confrontaciones políticas y la divergencia entre la teoría y la práctica política del sistema liberal, expuesta por J. Marcuello ${ }^{29}$, se puede entender como una constante no sólo política, sino una práctica social aplicable a la administración española. Se legisla y se planifica, pero ni se conocen ni se cum-

\footnotetext{
régimen liberal, parte del clero había apoyado la causa carlista; la desamortización de los bienes eclesiásticos había provocado una ruptura institucional. Por el nuevo acuerdo, el clero mantenía un estatuto peculiar en lo concerniente a los delitos civiles y criminales por lo que era necesario que el Ministerio de Gracia y Justicia conociera su número, situación y localización por motivos jurídicos.

26 G.M. 5-Noviembre-1856, n. ${ }^{\circ}$ 1402, relativa a la Comisión Estadística. R.D. 27-noviembre de 1856. G.M. 29 de Noviembre-1856. n. ${ }^{\circ} 1426$ relativa al Reglamento, normas y sistemas de provisión de plazas en la Estadística General.

27 Ibidem.

${ }^{28}$ Op. Cit. . R. Le Mée. (1979)

29 Marcuello, J. La práctica parlamentaria en el reinado de Isabel II. Madrid. Congreso de los Diputados. 1986.
} 
ple las normativas vigentes. Los deseos de modernidad no van acordes con la realidad cultural ni social de la mayoría de la población.

Desde 1808 y $1812^{30}$, las transformaciones derivadas de la abolición de los privilegios feudales, unido a los principios de igualdad de todos los habitantes a la hora de ser catalogado en el censo, modificaban la estructura de los censos anteriores, que habían sido realizados en función de su estamento, de las exenciones fiscales y de la clasificación del clero; en las nuevas encuestas se suprimían tales diferencias. La existencia de elecciones políticas necesitaba de una actividad censal precisa. En $1813^{31}$, las Cortes de Cádiz impusieron la contribución única, ordenaron a las Diputaciones Provinciales la obligación de un Censo de Población y la formación de la llamada «Estadística de la Provincia». La firma del Tratado de Valençay en 1813 y el regreso de Fernando VII cortó el desarrollo de estos planes censales.

Durante el Trienio Constitucional, la Ley del 3 de Febrero de 1823 ordenó la elaboración de un censo que no se realizó; las mismas bases establecidas, fueron de nuevo aprobadas en 1837, pero tampoco se aplicaron ${ }^{32}$, por lo que se recurría sistemáticamente al sistema de información de los Ayuntamientos y parroquias.

En la primera mitad del siglo XIX, no hubo un censo oficial, aunque se utilizara dicho término para designar los recuentos parciales de población que se fueron haciendo en el ámbito provincial: eran las llamadas Imputaciones provinciales, mediante las cuales se pedía a las provincias una información de los nacimientos y defunciones y con esta información se elaboraba el recuento total de población ${ }^{33}$. Estos datos, considerados oficiales, eran publicados en los Anuarios de estadística o Boletines oficiales. Existían otro tipo de organismos públicos que informaban de la población, como la Guía del Ministerio de Gobernación de 1836, del que sólo se publicó un número con la población de los Partidos Judiciales y la Matrícula Catastral de 1842, en la que figuraban datos de todos los Municipios.

La Imputación de 1817, dirigida por el Ministerio de Hacienda de Martín de Garay, tenía una finalidad tributaria; los trabajos de los intendentes para formar la Estadística General de España, con los consiguientes datos de población no llegaron a realizarse.

La Imputación de $\mathbf{1 8 2 2}^{34}$, realizada durante el Trienio Liberal, es elaborada por mandato de Cortes a partir del proyecto de reforma territorial sin considerar las

30 El estatuto de Bayona de 1808 y la Constitución de 1812 introdujeron el concepto de ciudadanía, aunque sólo afectara a la población masculina; con ello quedaban abolidas las exenciones fiscales y las diferencias estamentales.

31 Disposiciones de Cortes del 23 de Junio de 1813.

32 R.D. 29 DE Junio 1837. (G.M. 1-Julio- 1837, n. ${ }^{\circ}$ 942) con referencia la Ley de 3 de Febrero de 1823 como base del nuevo censo.

${ }^{33}$ García EspañA, E. «Imputaciones de población en la primera mitad del siglo XIX». En Revista de Fuentes Estadísticas. N. ${ }^{\circ}$ 50. Enero 2001.en www.//Demografía/revista\%20Fuentes\%20Estadísticas.htm

34 Decreto de Cortes de 12 de enero. Gaceta. 14 de enero de 1822. Se organizan los límites de las provincias y con ello la adscripción de los ayuntamientos. 
La actividad censal en España durante la primera mitad del siglo xIx en el contexto...

\begin{tabular}{|c|c|c|c|c|}
\hline \multicolumn{5}{|c|}{ POBLACIONES IMPUTADAS EN 1822, 1826, 1831 Y 1833.} \\
\hline & Año 1822 & 1826 & 1831 & 1832 \\
\hline \multicolumn{5}{|c|}{ VALENCIA : Alicante, Castellón, Játiva y Valencia. } \\
\hline Población & 965.930 & 1.256 .095 & 839.130 & 845.889 \\
\hline \multicolumn{5}{|c|}{$\begin{array}{l}\text { ANDALUCíA: Algeciras, Almería, Cádiz, Córdoba, Granada, Huelva, Jaén, Málaga, } \\
\text { Sevilla, Ceuta }\end{array}$} \\
\hline Población: & 2270644 & 2671321 & 2148553 & 2164075 \\
\hline \multicolumn{5}{|c|}{$\begin{array}{l}\text { CASTILLA LA VIEJA: Ávila, Burgos, León, Palencia, Salamanca, Segovia, Soria, } \\
\text { Valladolid, Villafranca, Zamora, Toro, Logroño, Santander. }\end{array}$} \\
\hline Población & 1.874 .722 & 2.455 .385 & 1.675 .243 & 1.594 .694 \\
\hline \multicolumn{5}{|c|}{ EXTREMADURA: Badajoz, Cáceres } \\
\hline Población & 500.430 & 556.780 & 490.612 & 484.359 \\
\hline BALEARES: & 229.093 & 242.893 & 214.003 & 214.836 \\
\hline \multicolumn{5}{|c|}{ CATALUÑA: Barcelona, Gerona, Lérida, Tarragona } \\
\hline Población & 909.315 & 1.116 .461 & 1.024 .122 & 1.035 .547 \\
\hline \multicolumn{5}{|c|}{ VASCONGADAS: Bilbao, San Sebastián, Vitoria } \\
\hline Población & 300.340 & 373.520 & 292.136 & 287.686 \\
\hline PAMPLONA & 195.416 & 288.244 & 260.520 & 264.477 \\
\hline \multicolumn{5}{|c|}{ ARAGÓN: Calatayud, Huesca, Teruel, Zaragoza } \\
\hline Población & 709.094 & 856.219 & 638.566 & 626.407 \\
\hline CANARIAS & 215.106 & & 200.534 & 200.534 \\
\hline \multicolumn{5}{|c|}{$\begin{array}{l}\text { CASTILLA LA NUEVA: Chinchilla, Ciudad Real, Cuenca, Guadalajara, Toledo, } \\
\text { La Mancha, Madrid }\end{array}$} \\
\hline Población. & 1.595 .055 & 1.580 .140 & 1.268 .983 & 1.281 .189 \\
\hline MURCIA ${ }^{35}$ & 253.370 & 493.192 & 438.693 & 443.063 \\
\hline \multicolumn{5}{|c|}{ GALICIA: Coruña, Lugo, Orense, Vigo ${ }^{36}$} \\
\hline Población & 1.267 .845 & 1.585 .419 & 1.344 .695 & 1.344 .695 \\
\hline OVIEDO ${ }^{37}$ & 375.505 & 464.565 & 371.849 & 370.823 \\
\hline TOTAL & 11.661 .865 & 13.940 .234 & 11.207.639 & 11.158 .274 \\
\hline
\end{tabular}

Cuadro n. ${ }^{\circ}$ 1. Estado de la población de 1822 a 1833. Fuente: Imputaciones originales. I.N.E.

35 La población de Chinchilla, pasa a partir del censo de 1826 a unirse a Murcia.

36 En la Imputación de 1822, Vigo es considerada provincia, en lugar de Pontevedra.

37 Hasta 1833, se considera Oviedo como Provincia; a partir de 1833 queda delimitada la Provincia de Asturias, con capital en Oviedo. (R.D. 30-11-1833) G.M. 3 de Diciembre. 
circunscripciones territoriales del siglo XVIII, ni tampoco las provincias consideradas “constitucionales» según el Art.10 de la Constitución de 1812. El Decreto 12-11822 dividía a España en cuenta 52 provincias; considerando como tales a Játiva (Valencia), individualizaba a Zaragoza, Bilbao, Villafranca (León) Calatayud, Chinchilla (Albacete), Pamplona, San Sebastián, Vigo y Vitoria.

La Imputación de 1826, al igual que las de 1831 y 1832, fueron realizadas en plena reacción absolutista ${ }^{38}$. El Duque del Infantado(1777-1841) ${ }^{39}$, López Ballesteros $^{40}$ y Tadeo Calomarde ${ }^{41}$ encargaron a la policía del recuento de población. Se consideraron 32 provincias y la integración de provincias en regiones.

Las Imputaciones de 1831 y 1832 fueron realizadas por la policía en una situación de turbulencia política: levantamientos políticos de Espoz y Minas en Francia(1830), el de Torrijos en Gibraltar(1831) y por otra parte, las rivalidades carlistas con respecto a las actuaciones de Calomarde, el conde Alcudia y el Obispo Abarca. En ese contexto, las imputaciones llevadas a cabo por la propia policía no fueran muy correctas. En 1831 y 1832 se consideran a Algeciras, Cádiz, Málaga, Granada y Ceuta como provincias independientes; se vuelve a utilizar el término de Santander y desaparece Toro. Se consideraron 33 provincias. En 1831 se informa de una población de 11.207.639 y en 1832 de 11.158.274.

Las Imputaciones de 1833 van asociadas a la reforma administrativa provincial. Al morir Fernando VII (29-IX-1833), la reina Gobernadora confirmó en el Gobierno a F. Cea Bermúdez y a Javier de Burgos como Secretario de Fomento. Dos meses después se promulgaba el Real Decreto por el que se disponía la división provincial de España en 49 provincias ${ }^{42}$, que «tomarán el nombre de sus respectivas capitales, excepto las de Navarra, Álava, Guipúzcoa y Vizcaya, que conservarán sus actuales denominaciones ${ }^{43}$ ». De este modo entendemos que hay un concepto claro entre la división administrativa moderna: la provincia, a la que quedarán referidos todos los censos demográficos y datos de carácter económico y el mantenimiento de la tradición de los antiguos reinos organizados en en regiones que se ha mantenido hasta 1978 como figuran en el cuadro $n .^{\circ} 2$.

${ }^{38}$ Con la intervención de las tropas francesas y los llamados 100.000 Hijos de san Luis, en realidad 95.000, se reimpuso el absolutismo más severo del reinado de Fernando VII, desde el 1-X-1823 al 11-XII1829.

${ }^{39} \mathrm{P}$. Alcántara Álvarez de Toledo, Duque del Infantado, Ministro de Estado entre 1825-1826.

40 L. López Ballesteros estuvo a cargo de la Hacienda durante el reinado de Fernando VII; ocupó el cargo desde $2712 / 1823$ hasta $1710 / 1832$.

${ }^{41}$ BELTRÁN, M et alii. Aragoneses ilustres. Zaragoza. Caja de Ahorros de la Inmaculada. 1983. pág. 50. Tadeo CALOMARDE (1773-1842) ministro de Fernando VII. Encargado de toda la información política-social del momento, el conocimiento del estado de la población era fundamental para su función política desde el Ministerio de Gracia y Justicia.

${ }^{42}$ GARCIA, E, Op. Cit. Pág. 3-4. Señala las pequeñas modificaciones de límites provinciales: en 1836, San Vicente de Alcántara, pasa de Cáceres a Badajoz; Albayda, Gandía y Onteniente con un total de 57 municipios pasan de Alicante a Valencia; Villena de Albacete y Sax, de Murcia, pasan a Jaén.

43. Art.1. ${ }^{\circ}$ R.D. del 30-11-1833 Estableció la actual división provincial de España. G. M.3/12/1833 
«Esta división de provincias no se entenderá limitada al orden administrativo, sino que se arreglarán á ellas las demarcaciones militares, judiciales y de la hacienda. ${ }^{44}$

Se marcaron los límites geográficos de cada provincia, según los acuerdos de las respectivas audiencias. Con posterioridad, en 1834, se subdividieron las provincias en partidos judiciales ${ }^{45}$; la finalidad era la regulación de la administración de justicia y servicios de intendencias que en definitiva respondían al principio constitucional de 1812, aunque dicha constitución no estuviera vigente. Se configuraron tres tipos de divisiones administrativas: la civil, anteriormente expuesta, la división militar y la eclesiástica. La división militar quedaba organizada en las Capitanías Generales de Andalucía, Aragón, Asturias, Canarias, Cataluña, Castilla la Nueva, Castilla la Vieja, Costa de granada, Extremadura, Galicia, Guipúzcoa, Mallorca, Navarra y Valencia. La jurisdicción eclesiástica se mantenía según las archidiócesis de Burgos, Granada, Santiago de Compostela, Sevilla, Tarragona, Toledo y Zaragoza, con los obispados exentos de Oviedo y León.

\begin{tabular}{|c|c|c|c|c|}
\hline \multicolumn{5}{|c|}{ DIVISIÓN PROVINCIAL DE ESPAÑA. R.D. 30-11-1833. } \\
\hline \multicolumn{2}{|c|}{ Provincias y regiones en 1833.} & \multirow[t]{2}{*}{ Población } & \multirow{2}{*}{\multicolumn{2}{|c|}{$\begin{array}{l}\text { C. Autónomas 1978-1983 } \\
\text { Población Absoluta y } \\
\text { porcentaje en } 1833 .\end{array}$}} \\
\hline Provincias & Región & & & \\
\hline Álava & \multirow{4}{*}{ Sin nombre expreso } & 67.523 & \multicolumn{2}{|c|}{ VASCONGADAS } \\
\hline Guipúzcoa & & 108.569 & \multirow[t]{2}{*}{287.530} & \multirow[t]{2}{*}{$2,34 \%$} \\
\hline Vizcaya & & 111.438 & & \\
\hline Navarra & & 230.925 & 230.925 & $1,9 \%$ \\
\hline Oviedo & ASTURIAS & 434.635 & 434.635 & $3,53 \%$ \\
\hline Murcia & \multirow{2}{*}{ MURCIA } & 283.540 & 283.540 & $2,32 \%$ \\
\hline Albacete & & 190.766 & \multicolumn{2}{|c|}{ CASTILLA LA MANCHA. } \\
\hline Ciudad Real & \multirow{5}{*}{ CASTILLA LA NUEVA } & 277.788 & \multirow{4}{*}{1.244 .708} & \multirow{4}{*}{$10,13 \%$} \\
\hline Guadalajara & & 159.375 & & \\
\hline Cuenca & & 334.582 & & \\
\hline Toledo & & 282.197 & & \\
\hline Madrid & & 320.000 & 320.000 & $2,60 \%$ \\
\hline
\end{tabular}

44 Ibidem. Art.4.

45 R. D. 18 de abril de 1834. G.M.23/4/1834. 


\begin{tabular}{|c|c|c|c|c|}
\hline Alicante & \multirow{3}{*}{ VALENCIA } & 368.961 & \multicolumn{2}{|l|}{ VALENCIA } \\
\hline Castellón & & 199.220 & \multirow[t]{2}{*}{957.142} & \multirow[t]{2}{*}{$7,79 \%$} \\
\hline Valencia & & 388.961 & & \\
\hline Almería & \multirow{8}{*}{ ANDALUCÍA } & 234.789 & \multirow{4}{*}{\multicolumn{2}{|c|}{ ANDALUCÍA. }} \\
\hline Cádiz & & 324.703 & & \\
\hline Córdoba & & 315.459 & & \\
\hline Granada & & 370.974 & & \\
\hline Huelva & & 133.470 & \multirow[t]{4}{*}{2.404 .132} & \multirow[t]{4}{*}{$9,56 \%$} \\
\hline Jaén & & 266.919 & & \\
\hline Málaga & & 390.515 & & \\
\hline Sevilla & & 367.303 & & \\
\hline Santander & \multirow{8}{*}{ CASTILLA LA VIEJA } & 169.057 & 169.057 & $1,37 \%$ \\
\hline Logroño & & 147.718 & 147.718 & $1,20 \%$ \\
\hline Ávila & & 137.903 & \multirow{3}{*}{\multicolumn{2}{|c|}{ CASTILLA-LEÓN. }} \\
\hline Burgos & & 224.407 & & \\
\hline Palencia & & 148.491 & & \\
\hline Segovia & & 134.854 & \multirow[t]{6}{*}{1.583 .098} & \multirow[t]{6}{*}{$12,89 \%$} \\
\hline Soria & & 115.619 & & \\
\hline Valladolid & & 184.647 & & \\
\hline León & \multirow{3}{*}{ LEÓN } & 267.438 & & \\
\hline Salamanca & & 210.314 & & \\
\hline Zamora & & 159.425 & & \\
\hline Badajoz & \multirow{2}{*}{ EXTREMADURA } & 306.092 & \multicolumn{2}{|c|}{ EXTREMADURA } \\
\hline Cáceres & & 241.328 & 547.420 & $4,45 \%$ \\
\hline I. Baleares & BALEARES & 229.197 & 229.197 & $1,86 \%$ \\
\hline Barcelona & \multirow{4}{*}{ CATALUÑA } & 442.273 & \multirow{2}{*}{\multicolumn{2}{|c|}{ CATALUÑA }} \\
\hline Gerona & & 214.150 & & \\
\hline Lérida & & 151.322 & \multirow[t]{2}{*}{1.041 .222} & \multirow[t]{2}{*}{$8,47 \%$} \\
\hline Tarragona & & 233.477 & & \\
\hline Canarias & CANARIAS & 229.197 & 199.950 & $1,62 \%$ \\
\hline
\end{tabular}


La actividad censal en España durante la primera mitad del siglo xIX en el contexto...

\begin{tabular}{|c|c|c|c|c|}
\hline Coruña & \multirow{4}{*}{ GALICIA } & 435.670 & \multirow{2}{*}{\multicolumn{2}{|c|}{ GALICIA }} \\
\hline Lugo & & 357.272 & & \\
\hline Orense & & 319.038 & 1.471 .982 & $12 \%$ \\
\hline Pontevedra & & 360.002 & & \\
\hline Huesca & \multirow{3}{*}{ ARAGÓN } & 214.874 & ARAGÓN & \\
\hline Teruel & & 218.403 & \multirow[t]{2}{*}{734.685} & \multirow[t]{2}{*}{$5,97 \%$} \\
\hline Zaragoza & & 301.408 & & \\
\hline TOTAL & \multicolumn{4}{|c|}{12.286 .941} \\
\hline
\end{tabular}

Cuadro n. ${ }^{\circ}$ 2. Población española según el R.D.30-11-1833.

En la Imputación de 1834, realizada por R D. 21-IV-1834, se contabilizaron 12.162.172 habitantes ${ }^{46}$, sirviendo de base para la elaboración de las elecciones a Cortes de 1836.

La Imputación de 1836, al igual que las posteriores de 1841, 1842, 1843, 1844 y 1846 se realizaron subdividiendo las provincias en los partidos judiciales con fines eminentemente electorales, dirigidas por el Ministerio de la Gobernación, y ejecutadas por los Corregidores y Alcaldes Mayores de los Ayuntamientos. El pseudo-censo queda ya configurado en partidos judiciales; eran momentos de «transición», porque mantiene el concepto tradicional de «vecino» y el equivalente en habitantes, así como el número de electores contribuyente.

Durante el Gobierno de José M. ${ }^{a}$ Calatrava ${ }^{47}$, por el R.D. 29 de junio de 1837 se ordenó la elaboración de un Censo en un momento álgido, coincidente con la aplicación del proceso desamortizador de Mendizábal ${ }^{48}$ y la elaboración de la Constitución de 1837. El censo decretado era de tipo moderno, con la misma técnica y datos para todo el territorio nacional y en fechas concertadas para la inscripción directa de los habitantes. La descripción del censo, con noventa artículos, se adelantaba a las instrucciones que posteriormente se admitieron en 1860, en el IV Reunión del Congrés Internacional de Statisque de Londres. Las guerras carlistas y la falta de recursos económicos paralizó este proyecto. El proyectado censo proyectado de 1837 no se realizó. Con motivo de las elecciones de 1837 se volvió a utilizar el sistema de imputaciones, con la cifra de 12.162.872 habitantes.

46 G.M. 22/Abril/1834. En el R. D. De 21 de Abril de 1834, se estableció la división de partidos judiciales, las provincias de 1833 repiten la misma cifra de población; son las mismas que se emplearon en el R.D. 21 -VIII-1836 para la elaboración del censo electoral.

47 José M. a Calatrava. (1786-1876) Ministro de Haciende durante la regencia de Espartero. RuLL SABATER, A. Diccionario sucinto de Ministros de Hacienda. (s. XIX-XX). Madrid. Instituto de Estudios fiscales. 1991.Doc. 16. pág.168.

48 Juan Álvarez MendizÁBal. (1790-1853) García Tejero, Alfonso. Historia político-administrativa de Mendizábal: dedicada al pueblo liberal español. Madrid. Tipografía Ortigosa. 1858. 
Terminada la guerra carlista, el Ministro de la Gobernación, Miguel Cortina, ordenó la formación del Resumen General de la Población y Utilidades de la Provincia $^{49}$. De nuevo, el Estado se valía de los Registros Parroquiales, como precursores del Registro Civil, durante la década de 1840-1850; los primeros libros de Movimiento Natural de la Población se basaron en datos de Registros Parroquiales. A partir de la Regencia de Espartero ${ }^{50}$ y durante toda la década de los años cuarenta, tenemos varias informaciones demográficas

La Imputación de 1841 difícilmente puede ser tenida en cuenta; recién nombrado el General Espartero como Regente, el Ministro de la Gobernación ${ }^{51}$ ordenó la realización de recogida de datos demográficos y económicos; sólo Madrid proporcionó los datos y 13 provincias ni contestaron ${ }^{52}$.

La Imputación de 1842 fue la base de la Matrícula Catastral ${ }^{53}$. A propuesta del Ministro de Hacienda, el 11 de julio se ordenó la recogida de datos que una vez recibidos, se agruparon bajo tal nombre. El recuento de población era inferior a las anteriores, de 11.712.460 habitantes. Madoz ${ }^{54}$ analiza los datos, los estudia, considera que no son correctos según las informaciones que él tenía y desecha la cifra de población, pero no descarta la organización del sistema de contabilidad del que posteriormente se sirve.

La Imputación de 1843 fue una continuidad de los trabajos iniciados y rectificados en 1842. Los datos fueron reproducidos en el Censo de Madoz ${ }^{55}$. Su realización fue llevada a cabo por el Ministerio de Gracia y Justicia dirigido por Joaquín María López.

La Imputación de $\mathbf{1 8 4 4}$ se realizó según los datos de los Registros Municipales. Dado el interés del Gobierno de Narváez y del ministro de la Gobernación, Pedro José Pidal, en preparar la Ley de Administración Local, se centró en la población según municipios acorde con la legislación ya existente desde 1835 . El temor que este recuento de habitantes por municipios implicara una subida de im-

49 D. 7-2-1841. por el que se ordena la formación de Juntas Provinciales para la contabilización de la población. Según Madoz, los resultados están infravalorados y de hecho trece provincias carecen de imputaciones.

50 Espada Burgos, M. Baldomero Espartero, un candidato al trono de España. Ciudad Real Diputación Provincial. 1984. Baldomero ESPARTERO (1793-1879) De un seminario para ser sacerdote, pasó a militar. De militar a regente y estadista.

51 G.M. El Decreto de 7 de febrero de 1841, el ministro de Gobernación, Manuel Cortina, a través de los Partidos Judiciales, se ordena a los Intendentes que elaboren una memoria con los datos demográficos y económicos de cada provincia.

52 García España, E. Poblaciones imputadas en la primera mitad del siglo XIX. I.N.E. Madrid.2002. P.135.

53 La llamada Matrícula catastral, es la recolección de los datos demográficos, decretada por el Presidente de Gobierno, Marqués de Redil, a petición del Ministro de Hacienda, Ramón M. ${ }^{\text {a }}$ Calatrava, el 11 de Julio de 1842.

${ }_{54}$ Madoz. Diccionario Geográfico, Estadístico, Histórico de España y sus posesiones. Madrid. 1850.

55 Pascual Madoz. (1806-1880). Publicista, político, diputado, gobernador civil y Ministro, en su obra científica y en su labor de gobierno impulsa la formalización de los censos. 
La actividad censal en España durante la primera mitad del siglo xIX en el contexto...

\begin{tabular}{|c|c|c|c|}
\hline Años & Organismo & Total Nacional & Extremadura \\
\hline 1822 & Decreto de Cortes & 11.661 .865 & $4,29 \%$ \\
\hline 1826 & Policía & 13.940 .234 & $3,99 \%$ \\
\hline 1831 & Policía & 11.207 .639 & $4,38 \%$ \\
\hline 1832 & Policía & 11.158 .174 & $4,34 \%$ \\
\hline 1833 & M. ${ }^{\circ}$ Fomento. & 12.286 .941 & $4,46 \%$ \\
\hline 1836 & M. ${ }^{\circ}$ Gobernación & 11.800 .413 & $4,42 \%$ \\
\hline 1837 & Elecciones Cortes & 12.162 .872 & $4,50 \%$ \\
\hline 1842 & Matrícula Catastro & 11.812 .445 & $5,30 \%$ \\
\hline 1843 & Justicia/ Madoz & 14.216 .219 & $4,23 \%$ \\
\hline 844 & Registro Municipal & 11.298 .098 & $5,35 \%$ \\
\hline 1846 & Gobernación & 11.993 .834 & $4,66 \%$ \\
\hline 1850 & Gobernación & 10.942 .280 & $4,79 \%$ \\
\hline
\end{tabular}

Cuadro. N. ${ }^{\circ}$ 3. Población de España según las Imputaciones oficiales. I.N.E y porcentaje de población de Extremadura.

puestos, es lo que pudiera explicar que el descenso de habitantes no se deba a la realidad, sino a un ocultamiento de información.

Las imputaciones de $1846^{56}$ tenían como finalidad básica la organización de las elecciones y la formación de los distritos electorales, no siempre coincidentes con los partidos judiciales. Refieren una población de 12.993.734; mantienen las mismas provincias con algunos cambios de adscripción municipal ${ }^{57}$. De acuerdo a los datos demográficos, recogidos de la Ley electoral (18-3-1846), se establecieron el número de diputados en Cortes que pertenecían a cada provincia, con un total de 349 escaños. Solamente se modificaron los datos de diez provincias, por lo que se deduce que en realidad lo que se hizo fue una revisión de las Imputaciones anteriores; quedaron sin integrar Ceuta y Melilla.

Las Imputaciones de 1850 contabilizan 10.942.280 habitantes. Es la última imputación oficial. Según García Fuentes ${ }^{58}$, es una cifra poco fiable porque supone el $70 \%$ de la población del censo oficial de 1857; a esto creo que habría que añadir el hecho de que no hay una crisis demográfica fuerte, ni momentos de carestía, que explique un descenso de dos millones de habitantes con respecto al anterior y cinco millones con el posterior.

56 Los datos de población vienen anexadas a la Ley del 24 de Marzo de 1846, por la que se regulaba el número de diputados y distritos electorales.

57 En 1.845, Oñate, municipio exento, se adscribió a Guipúzcoa; en 1846 Villarrobledo pasa de Ciudad Real a Albacete.

58 Ibidem. 


\section{INFORMACIONES DEMOGRÁFICAS DE CARÁCTER CIENTÍFICO Y PARTICULAR}

A lo largo del siglo, la particular trayectoria de pugna política, generó dos ámbitos de información demográfica, la oficial y la de carácter particular. En algunos casos, liberales, exiliados en el extranjero, en otros casos, eruditos sacan a la luz informaciones históricas. José Canga Argüelles (1770-1843) publicó el Diccionario de Hacienda en Londres (1826-1827), reseña estadística, en la que se incluía la división territorial, política y población. Tomás González, sacaba a la luz en 1829, del Archivo de Simancas, los Censos de la población de las provincias y partidos de la Corona de Castilla en el siglo XVII. Sebastián Miñano Bedoya (1770-1840) publicó en 1826 el Diccionario Geográfico-Estadístico de España y Portugaª, en donde recoge toda la información geográfica de todas las provincias españolas.

La obra más significativa fue la de P. Madoz; a su regreso del exilio publicó la obra de Moreau de Jonnes, Estadísticas en España ${ }^{60}$; la obra fue muy bien recibida, se vendió con éxito, por lo que inició la impresión del Diccionario Geográfico, estadístico e Histórico ${ }^{61}$, publicado entre 1833 y 1845. Madoz, como responsable de la comisión Estadística, contó con la información demográfica del Ministerio de Gobernación. Simultáneamente, en 1844, la Sociedad Económica Matritense, abrió una Cátedra de Estadística con 39 alumnos encomendada a D. José M. ${ }^{a}$ Ibáñez, Secretario de la Comisión de Estadística cuyo presidente era D. Pascual Madoz; aportación importantísima ya que en muchos casos se conocen los datos del censo de Aranda por estar contenidos en su obra; de igual manera queda perfectamente acotados los términos de la administración de Provincia, audiencia, intendencia, vicaría, partido y villa. En 1844, Fermín Caballero publicaba el Manual geográfico-administrativo de la Monarquía Española ${ }^{62}$, donde se daban datos demográficos, económicos, mercantiles y demás informaciones que evidencia la adaptación del sistema de medidas, pesos, moneda tradicional al sistema europeo.

\section{EL CRECIMIENTO DE LA POBLACIÓN EN LA PRIMERA MITAD DEL SIGLO XIX}

No hubo censos generales de población, pero sí una permanente información sobre la misma, realizada por diversos organismos, con distintas finalidades y con grandes diferencias en cuanto a su fiabilidad según las distintas provincias. La

59 El Diccionario fue publicado a lo largo de los años de 1826-29 por la Empresa hispano francesa Pierrot-Peralta.

60 Moreau de Jonnes, Statisque de l'Espagne. París 1834

61 P. MADOz. Diccionario geográfico-estadístico-histórico de España y sus posesiones de ultramar. Madrid. Tipografía del Diccionario. 1846-1850.

62 F. CABALleRo. Manual geográfico-administrativo de la Monarquía española.. con 1750 artículos en orden alfabético. Madrid. Madrid. Antonio Jenes, 1844. Recoge una información de las poblaciones españoles. BN. 
poca veracidad de los datos, ha dado lugar a que se establezcan índices correctores en función de los datos del censo oficial de 1857, estableciendo unas estimaciones más acordes con la realidad como nos muestra el gráfico n. ${ }^{\circ} 1$.

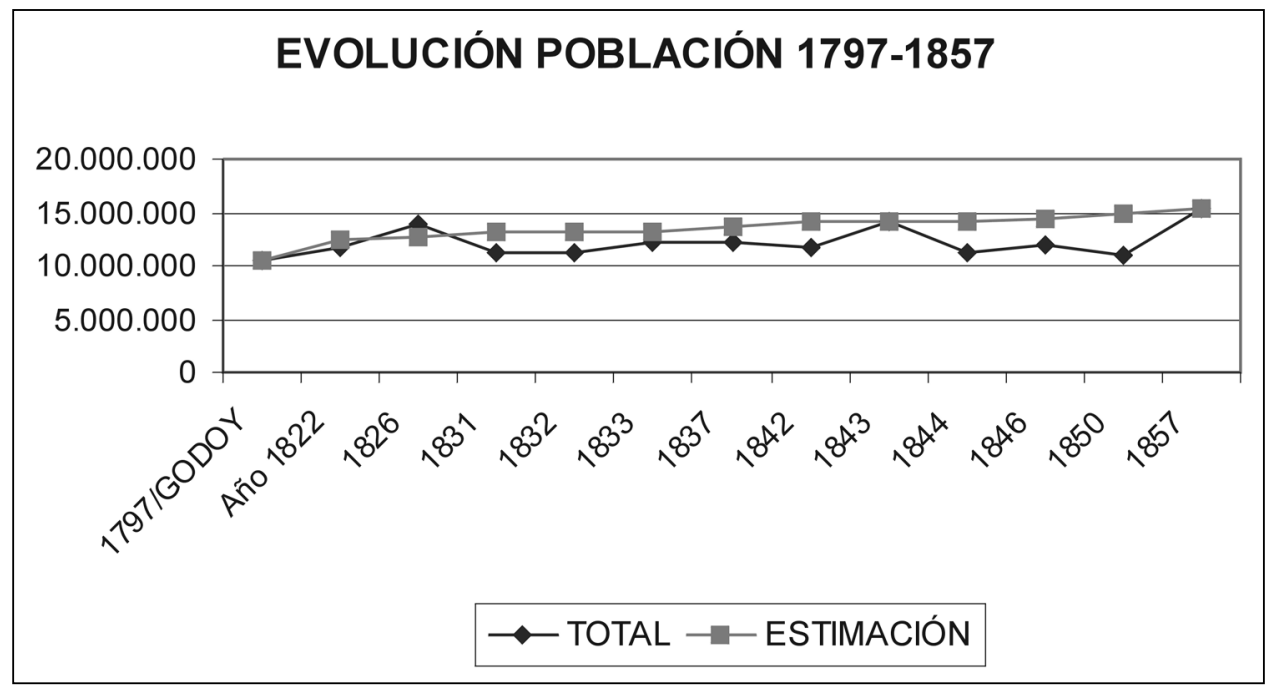

Gráfico n. ${ }^{\circ}$ 1. Fuente: datos oficiales contrastados con los cálculos estimativos de corrección de E. García España. I.N.E

La oscilación de la población de uno a otro año, manifiesta las irregularidades a las que estuvieron sometidos. Consideramos que las cifras son demasiado aleatorias y poco fidedignas, donde crecimiento y descenso de la población no tienen una justificación lógica, salvo las provincias directamente afectadas por la guerra civil carlista. Por otra parte, las crisis de subsistencias en 1817, 1823-25 y 1837, señaladas por N. Sánchez Albornoz ${ }^{63}$, no parecen reflejarse en los datos demográficos generales.

En la distribución de la población por regiones (Gráfico n. ${ }^{\circ}$ 2) según la territorialización emanada de la nueva organización administrativa de 1833, observamos diferentes ritmos de crecimiento: Cantabria, Castilla-León, País Vasco, Castilla-La Mancha y La Rioja, redujeron su proporcionalidad en 1 punto en el total nacional; Navarra, Aragón, Valencia, Baleares, Murcia y Canarias mantienen la misma proporción; Galicia, Asturias, Cataluña, Madrid, Extremadura y Andalucía aumentaron su proporción en 1 punto en el total nacional, por lo tanto, eran estas regiones las que entre 1822-1846, momento de transición política y transformación económica, mantuvieron mayor vitalidad demográfica

63 SÁnchez Albornoz. La crisis de subsistencias de España en el siglo XIX. Rosario. Argentina. Instituto de Investigaciones Históricas 


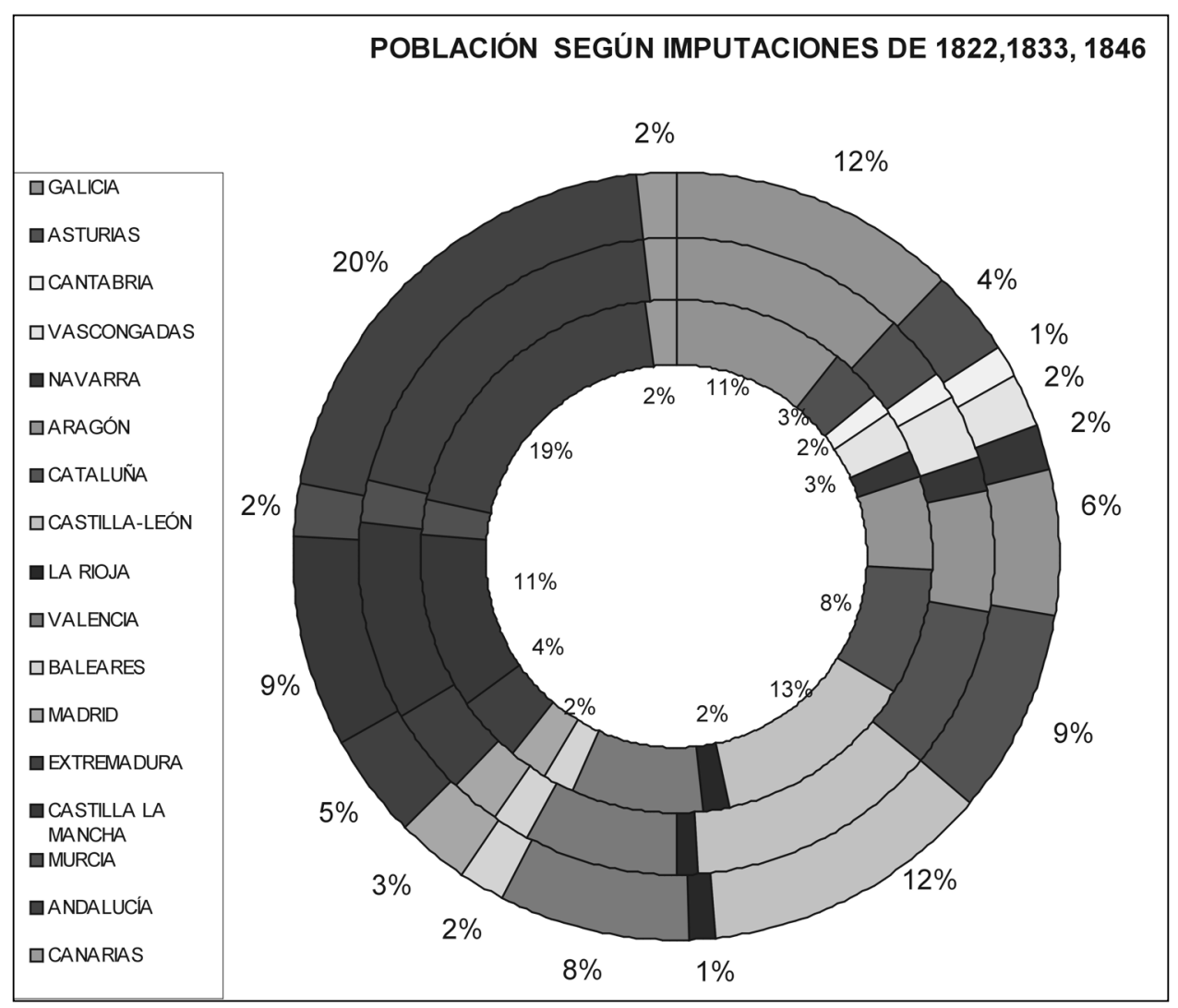

Gráfico n. ${ }^{\circ}$. Población contrastada de los datos de las imputaciones de 1822, línea interior, 1833 y 1846, línea exterior. Adaptación de los datos a las Comunidades Autónomas derivadas de la Constitución de 1978. Fuente: I.N.E. Anotamos los porcentajes cuando hay diferencias entre 1822 y 1846.

En cuanto al crecimiento anual, en proporción constante a la tasa de crecimiento anual, aceptando de antemano la poca fiabilidad de los datos, observamos que no se constatan cambios, pero si hay diferencias regionales. Cantabria, País vasco, Castilla-León. La Rioja, Valencia, Castilla-La Mancha, Canarias presentan índices de crecimiento negativos, atribuidas a las epidemias generales de cólera de 1833-1835 según estudios de Sánchez Albornoz ${ }^{64}$.

Entre 1822-1846, según regiones históricas, el mayor crecimiento es el de Asturias y Extremadura que cuadruplica a la media de España. Si aceptamos el sistema originario de la formación de regiones, según el R.D.30.11.1833, de la división provincial, pero establecemos una periodicidad mayor y mantenemos la fiabilidad terminal de referencia del censo de 1857, el resultado final sería diferente

64 Ibidem. 


\begin{tabular}{|l|r|l|r|}
\hline \multicolumn{4}{|c|}{ CRECIMIENTO INTERCENSAL 1822- 1846 } \\
\hline Oviedo/Asturias & 0,764 & Canarias & $-0,021$ \\
\hline Galicia & 0,602 & Valencia & $-0,080$ \\
\hline Pamplona(Navarra) & 0,528 & León & $-0,145$ \\
\hline Cataluña & 0,495 & Vascongadas & $-0,221$ \\
\hline Extremadura & 0,461 & Castilla la Vieja & $-0,336$ \\
\hline Andalucía & 0,236 & Castilla la Mancha & $-0,562$ \\
\hline Aragón & 0,146 & Murcia & $-0,568$ \\
\hline Baleares & 0,065 & MEDIA & $\mathbf{0 , 1 1 7}$ \\
\hline
\end{tabular}

Cuadro n. ${ }^{\circ}$. Tasas de crecimiento. Datos obtenidos de las Imputaciones de 1822 y 1846 . I.N.E.

al expresado según comunidades autónomas actuales, ya que las provincias cambian su configuración y también es diferente al establecido con carácter general por P. Vilar65. El aumento de población en cifras absolutas, fue positivo al pasar de 11.661.865 en 1822 a 15.116.299 según el censo oficial de 1857. Las regiones con mayor vitalidad demográfica, a pesar de las guerras carlistas fueron Asturias, Galicia, Cataluña, Navarra y Extremadura; la de menor crecimiento fue Castilla La Nueva, cuestión paradójica ya que incluía Madrid.

El crecimiento de España en la primera mitad de siglo XIX, contabilizando los datos del censo oficial de 1857, sería de un 0,8 con diferencias significativas por regiones:

\begin{tabular}{|l|c|l|l|}
\hline \multicolumn{4}{|c|}{ CRECIMIENTO INTERCENSAL 1822- 1857 } \\
\hline CATALUÑA & 1,721 & CASTILLA LA VIEJA & 0,752 \\
\hline NAVARRA & 1,207 & ANDALUCÍA & 0,738 \\
\hline EXTREMADURA & 0,993 & VALENCIA & 0,731 \\
\hline GALICIA & 0,969 & ARAGÓN & 0,621 \\
\hline OVIEDO/Asturias & 0,960 & BALEARES & 0,394 \\
\hline VASCONGADAS & 0,918 & CANARIAS & 0,241 \\
\hline LEÓN & 0,870 & CASTILLA-LA NUEVA & 0,137 \\
\hline MURCIA & 0,805 & MEDIA & 0,810 \\
\hline
\end{tabular}

Cuadro n. ${ }^{\circ}$ 5. Tasas de crecimiento de 1822-1857 según datos de las Imputaciones oficiales y Censo de 1857.

65 Op. Cit. 
Estos datos nos orienta sobre dos etapas de crecimiento en la primera mitad del siglo XIX, teniendo siempre presente que no hay fiabilidad absoluta de los datos; una primera, durante el primer tercio del siglo XIX, que se alargaría hasta 1840 en las zonas de conflicto carlista, y otra segunda fase de mayor crecimiento, a partir de 1830.

En relación con Europa, Livi-Bacci66, considerando un índice 100 en 1750, establece una comparación del crecimiento en diferentes países europeos en 1850. Mientras Inglaterra, a lo largo del siglo pasa a un índice de 289 , el resto de las naciones se mantienen entre 180 y 160, siendo el índice de 171 el que corresponde a España, lo cual nos indica que el comportamiento demográfico de la península en la primera mitad del siglo XIX, fue similar al de Italia, Francia, Holanda y Alemania.

\section{CONCLUSIONES.}

Durante la primera mitad del siglo hay un especial interés por crear los órganos e instrumentos adecuados para la realización de un censo moderno y fiables, objetivo que se trató de conseguir desde las diferentes posiciones del liberalismo e incluso desde el absolutismo del monarca borbónico. Se organizaron los servicios estadísticos y se creó un cuerpo de funcionarios especialistas. En este sentido la situación de España es similar a la de todos los Estados Europeos. A través de los Congresos Internacionales; se coordinaron las experiencias.

La realidad de los «dos Españas», la oficial y la real, también queda ejemplarizada en la ocultación de la población, era tan evidente, que se tuvo que recurrir a la policía para hacer el recuento de población. Las imputaciones de 1826, durante la etapa absolutista de Fernando VII, nos ofrece la situación demográfica más real del momento; otras imputaciones posteriores, carecen de fiabilidad: era imposible que de 1850 a 1857 , el crecimiento absoluto de la población fuera de cinco millones

El hecho más significativo fue el establecimiento de una normativa legislativa y una infraestructura institucional que culminaba en 1856, estableciendo las base legales de todos los censos posteriores. Si bien podemos considerar que se mantenía la etapa censal iniciada con el censo de Aranda de 1768, que denominamos protoestadística, a partir de 1856 podemos afirmar el inicio de la etapa científica, metódica y sistemática de los censos modernos. El paso hacia la modernización estaba consolidado desde el momento en que el Estado tenía instrumentos e instituciones especializadas en conocer la situación cuantitativa y cualitativa de todos los ciudadanos.

66 LIVI BACCI, Máximo, Ensayo sobre la Historia demográfica europea. Población y alimentación en Europa. Barcelona. Ariel. 1988. pág. 19. 


\section{FUENTES DOCUMENTALES Y BIBLIOGRÁFICAS.}

Bargueño Rivero, J. Geografía política de la España constitucional. La división provincial. Madrid. Centro de Estudios Constitucionales. 1996.

Beltrán, M et alii. Aragoneses ilustres. Zaragoza. Caja de Ahorros de la Inmaculada. 1983.

BertalanfFy, L. Teoría general de los sistemas. México. FCE. 1989

Burgos, J. Anales del Reinado de Isabel II. Madrid. Tipografía Mellado. 1850-51.v. 6

CABAllero, F. Manual geográfico-administrativo de la Monarquía española.. con 1750 artículos en orden alfabético. Madrid. Madrid. Antonio Jenes, 1844. FLORES ESTRADA, A. Plan para formar la Estadística en la provincia de Sevilla. Sevilla 1814. Madrid. Edición facsímile del I.N.E. 1983

Censo de la matrícula catastral. Revisado y completado por P. Madoz. Facsímile. Madrid. I.N.E. 2002.

Censo de la población de España de el año 1797. Ejecutado por orden del Rey en el 1801Madrid.1803 Edición facsímile INE. 1992

CENSO DE LA POBLACIÓN DE ESPAÑA. 1857. Madrid. Imprenta Nacional. 1858.

CONSTITUCIÓN de Cádiz, 1812. Constitución de 1837, Constitución de 1845., Proyecto de Constitución de 1856, Reformas Constitucionales de 1856 y 1857.

GACETA DE MADRID: Años : 1802, 1814, 1822, 1833, 1834, 1835, 1836, 1837, 1841, 1845, 1846, 1847, 1848, 1850, 1851, 1852, 1853, 1854, 1855,1856, 1857.

Garay, Martín de, Memoria presentada por Don Martín de Garay, Ministro del despacho de Hacienda, al Rey. Madrid. 1817

GARCíA ESPAÑA, E. «Imputaciones de población en la primera mitad del siglo XIX». En Revista de Fuentes Estadísticas. N. ${ }^{\circ} 50$. Enero 2001.

García TeJero, Alfonso. Historia político-administrativa de Mendizábal: dedicada al pueblo liberal español. Madrid. Tipografía Ortigosa. 1858.

GonzÁLEZ LóPEZ, E. Luís López Ballesteros (1782-1853).La Coruña. Fundación Pedro Barrie de la Maza. 1987.

LIVI BACCI, M, Ensayo sobre la Historia demográfica europea. Población y alimentación en Europa. Barcelona. Ariel. 1988.

Madoz, P. Diccionario geográfico-estadístico-histórico de España y sus posesiones de ultramar. Madrid. Tipografía del Diccionario. 1846-1850.

MARCuELLO, J. La práctica parlamentaria en el reinado de Isabel II. Madrid. Congreso de los Diputados. 1986.

MoREAU DE Jonnes, Statisque de l'Espagne. París 1834

POBLACIONES IMPUTADAS DE LAS ANTIGUAS PROVINCIAS.1822, 1826,1831, 1832, 1833, 1834,1837,1846,1850. Edición facsímile. Madrid. I.N.E. 2002.

Rull Sabater, A. Diccionario sucinto de Ministros de Hacienda. (s. XIX-XX. Madrid. Instituto de Estudios fiscales. 1991.Doc. 16. pág.168.

SAMPaIO, A.P.F. Elementos de la Ciencia estadística. Madrid, 1841.Edición facsímile. INE. Madrid. 1982

SÁnCHeZ Albornoz, N. La crisis de subsistencias de España en el siglo XIX. Rosario. Argentina. Instituto de Investigaciones Históricas

SÁNCHEZ-LAFUENTE. Historia de la estadística como ciencia (1500-1900). Madrid. Ministerio de Planificación y Desarrollo. 1975. pág. 107 y ss. 